\title{
Critical parameters and universal amplitude ratios of two-dimensional spin- $S$ Ising models using high- and low-temperature expansions
}

\author{
P. Butera* and M. Comi \\ Istituto Nazionale di Fisica Nucleare and \\ Dipartimento di Fisica, Università di Milano-Bicocca \\ 3 Piazza della Scienza, 20126 Milano, Italy \\ A. J. Guttmann ${ }^{\dagger}$ \\ Department of Mathematics and Statistics, \\ The University of Melbourne, Victoria, 3010, Australia.
}

(Dated: March 16, 2021)

\begin{abstract}
For the study of Ising models of general spin $S$ on the square lattice, we have combined our recently extended high-temperature expansions with the low-temperature expansions derived some time ago by Enting, Guttmann and Jensen. We have computed for the first time various critical parameters and improved the estimates of others. Moreover the properties of hyperscaling and of universality (spin $S$ independence) of exponents and of various dimensionless amplitude combinations have been verified accurately. Assuming the validity of the lattice-lattice scaling, from our estimates of critical amplitudes for the square lattice we have also obtained estimates of the corresponding amplitudes for the spin $S$ Ising model on the triangular, honeycomb, and kagomé lattices.
\end{abstract}

PACS numbers: PACS numbers: 05.50+q, 11.15.Ha, 64.60.Cn, 75.10.Hk

Keywords: Ising model, hyperscaling, universality, universal combinations of critical amplitudes, hightemperature expansions, low-temperature expansions, lattice-lattice scaling 


\section{INTRODUCTION}

The properties of the spin $S=1 / 2$ two-dimensional Ising model with nearest-neighbor interactions in zero magnetic field, have been extensively explored in the last six decades. Much more modest efforts have been devoted to the study of the simplest generalizations of the model to spin $S>1 / 2$. The main reason is probably that these models are not known to be solvable or, at least, to have any simple duality property which can help to extend the small body of information coming from numerical methods of limited accuracy such as stochastic simulations, series expansions or transfer-matrix calculations.

The first important result from a comparative study of Ising models for different values of the spin came from pioneering work by Domb and Sykes ${ }^{1}$. They analysed the high-temperature(HT) expansion of the susceptibility $\chi(K ; S)$ through $O\left(K^{6}\right)$ in the threedimensional case and conjectured that the value of the critical exponent $\gamma(S)$ is independent of the spin magnitude. This was the first step towards the modern formulation of the criticaluniversality hypothesis. Similar analyses were soon repeated by other authors using both $\mathrm{HT}^{2,3}$ and low-temperature (LT) expansions ${ }^{4}$ for two-dimensional systems. Unfortunately, the series derived in those years were rather short and therefore the results of the analyses could not reach a sufficient accuracy or were inconclusive. It was only in 1980 that Nickel ${ }^{5,6}$ finally extended through $O\left(K^{21}\right)$ the HT series in two dimensions on the square (sq) lattice and in three dimensions on the body-centered cubic lattice. The expansions of $\chi(K ; S)$ and of the second moment of the spin-spin correlation function $\mu_{2}(K ; S)$ were then published only for $S=1 / 2,1,2, \infty$. More recently also the LT expansions on the sq lattice for $S=1 / 2,1,3 / 2,2,5 / 2,3$ were considerably extended by Enting, Jensen and Guttmann ${ }^{7}$. We have summarized in Table I and Table II the state of $\mathrm{HT}^{3,8}$ and $\mathrm{LT}^{7}$ expansions before our work.

In spite of the very large number of LT expansion coefficients now available, the analysis of the series remains arduous due to occurrence ${ }^{7}$ of numerous unphysical singularities in the complex temperature plane ${ }^{9}$ which are closer to the origin than the physical singularity and whose structure becomes increasingly complicated with $S$. As a consequence, the LT study of Ref. ${ }^{7}$ has been an alarming lesson on the subtleties in the analysis of slowly convergent series more than a source of accurate estimates of the critical parameters of the models.

Many intriguing indications and conjectures about the structure of these unphysical singularities also came in the same period from work by Matveev and Shrock ${ }^{10}$ who examined

TABLE I: The longest HT expansions, published (or obtainable from data in the literature) before our work ${ }^{11,12}$, for the susceptibility $\chi(K ; S)$, the second moment of the correlation function $\mu_{2}(K ; S)$ and the second field-derivative of the susceptiblity $\chi_{4}(K ; S)$ in the case of the Ising models with general spin $S$ on two-dimensional lattices. It should be noted that in the special case $S=1 / 2$, on the sq lattice, much longer expansions ${ }^{13}$ for $\chi$ and $\mu_{2}$ have been computed. However, the published expansions $^{5}$ of $\chi_{4}(K ; 1 / 2)$ on the sq lattice do not extend beyond $K^{17}$.

\begin{tabular}{lccc}
\hline \hline Observable & Lattice & Order & Ref. \\
\hline$\chi(K ; S), \mu_{2}(K ; S)$ & $\mathrm{sq}$ & 21 & 6 \\
$\chi_{4}(K ; S)$ & $\mathrm{sq}$ & 10 & 8 \\
$\chi(K ; S), \mu_{2}(K ; S)$ & $\operatorname{tr}$ & 10 & 3 \\
$\chi_{4}(K ; S)$ & $\operatorname{tr}$ & 10 & 8 \\
\hline \hline
\end{tabular}


TABLE II: The longest LT expansions, presently available for the spontaneous magnetization, the specific heat and the susceptibility in the case of the Ising models with various values of the spin on the sq lattice.

\begin{tabular}{lccc}
\hline \hline Observable & Lattice & Order & Ref. \\
\hline$M(u ; 1), C(u ; 1), \chi(u ; 1)$ & $\mathrm{sq}$ & 113 & 7 \\
$M(u ; 3 / 2), C(u ; 3 / 2), \chi(u ; 3 / 2)$ & $\mathrm{sq}$ & 100 & 7 \\
$M(u ; 2), C(u ; 2), \chi(u ; 2)$ & $\mathrm{sq}$ & 119 & 7 \\
$M(u ; 5 / 2), C(u ; 5 / 2), \chi(u ; 5 / 2)$ & $\mathrm{sq}$ & 126 & 7 \\
$M(u ; 3), C(u ; 3), \chi(u ; 3)$ & $\mathrm{sq}$ & 154 & 7 \\
\hline \hline
\end{tabular}

the spin $S$ models on various two-dimensional lattices using transfer-matrix methods.

Here we discuss some results of an analysis of HT series for the sq lattice recently extended ${ }^{11}$ by linked-cluster expansion techniques.

For the nearest-neighbor correlation function $G(K ; S)$, for $\chi(K ; S)$, and $\mu_{2}(K ; S)$ our series reach order $K^{25}$, while for the second field derivative of the susceptibility $\chi_{4}(K ; S)$ they extend through $O\left(K^{23}\right)$. In order to make alternative analyses possible, our vast collection of series data both for two- and three-dimensional lattices was made easily accessible ${ }^{12}$ on-line for $S=1 / 2,1,3 / 2,2,5 / 2,3,7 / 2,4,5, \infty$. It should be noted that HT and LT expansions as extensive as those obtained by Nickel et al. in Ref. ${ }^{5}$ and more recently in Ref. ${ }^{13}$ (only for $\chi$ ) in the very special case of the (partially solvable) two-dimensional $S=1 / 2$ Ising model seem presently beyond reach for $S>1 / 2$.

The HT series show somewhat simpler and faster convergence properties than the LT series, because the behavior of the coefficients is dominated by the physical singularity. Although, even in this case, these favorable properties slightly deteriorate for $S>2$, we can hope to determine basic HT critical parameters with a reasonable accuracy for various values of $S$. Moreover it is also worthwhile to reconsider the LT expansions of Ref. ${ }^{7}$ for the sq lattice, because by relying on the results of our HT analysis, we can improve some estimates of the LT critical parameters and thus obtain new determinations of universal combinations ${ }^{14}$ of LT and HT amplitudes. No theoretical surprises are expected from this analysis, however we believe it is still useful to improve the rather modest numerical precision presently available even for basic critical parameters like the critical temperatures, to determine various critical amplitudes for which no estimates are yet known and to use our results to test with higher accuracy the validity of hyperscaling and of universality with respect to the magnitude of the spin.

Almost all the computational effort in extending series for the two-dimensional Ising model for $S>1 / 2$ has been devoted to square-lattice series. However by making use of the theory of lattice-lattice scaling, as developed by Betts et al. ${ }^{15}$ and extended by Gaunt and Guttmann $^{16}$, using our estimates of the critical amplitudes on the square lattice, we are able to calculate the corresponding amplitudes on other two-dimensional lattices to precisely the same precision as they are known for the square lattice.

\section{THE SPIN- $S$ ISING MODELS}

The spin- $S$ Ising models with nearest-neighbor interaction are defined by the Hamiltonian: 


$$
H\{s\}=-\frac{J}{2} \sum_{\left(\vec{x}, \vec{x}^{\prime}\right)} s(\vec{x}) s\left(\vec{x}^{\prime}\right)-h \sum_{\vec{x}} s(\vec{x})
$$

where $J$ is the exchange coupling, and $s(\vec{x})=s^{z}(\vec{x}) / S$ with $s^{z}(\vec{x})$ a classical spin variable at the lattice site $\vec{x}$, taking the $2 S+1$ values $-S,-S+1, \ldots, S-1, S$. The sum runs over all nearest-neighbor pairs of sites. We shall restrict ourselves to the square lattice and consider expansions either in the usual HT variable $K=J / k_{B} T$ and in the natural LT variable $u(S)=\exp \left(-K / S^{2}\right)$. Here $T$ is the temperature, $k_{B}$ the Boltzmann constant, and $K$ will be called "inverse temperature" for brevity. In the critical region we shall also refer to the standard reduced-temperature variable $t(S)=T / T_{c}(S)-1=K_{c}(S) / K-1$.

In the HT phase, the basic observables are the connected $2 n$-spin correlation functions. Our series ${ }^{12}$ cover quantities related to the two-spin correlation functions $\langle s(\vec{x}) s(\vec{y})\rangle_{c}$ and to the four-spin correlation functions $\langle s(\vec{x}) s(\vec{y}) s(\vec{z}) s(\vec{t})\rangle_{c}$.

In the LT phase the symmetry is broken and the $n$-spin correlations are non trivial also for odd $n$. In particular, we shall reconsider the LT expansions of the magnetization, the susceptibility and the specific heat derived for $S=1,3 / 2,2,5 / 2,3$ in Ref. ${ }^{7}$.

The spontaneous magnetization is defined by

$$
M(T ; S)=\lim _{h \rightarrow 0+}\langle s(\overrightarrow{0})\rangle .
$$

The internal energy per spin is given in terms of the nearest-neighbor correlation function by

$$
U(T ; S)=-\frac{q J}{2}\langle s(\overrightarrow{0}) s(\vec{\delta})\rangle
$$

where $\vec{\delta}$ is a nearest-neighbor lattice vector and $q$ is the lattice coordination number.

The specific heat is the temperature-derivative of the internal energy at fixed zero external field

$$
C_{H}(T ; S)=\frac{d U(T ; S)}{d T} .
$$

In terms of $\chi(T ; S)$, the zero-field reduced susceptibility,

$$
\chi(T ; S)=\sum_{\vec{x}}\langle s(\overrightarrow{0}) s(\vec{x})\rangle_{c}
$$

and of $\mu_{2}(T ; S)$, the second moment of the correlation function,

$$
\mu_{2}(T ; S)=\sum_{\vec{x}} \vec{x}^{2}\langle s(\overrightarrow{0}) s(\vec{x})\rangle_{c}
$$

the "second-moment correlation length" $\xi(T ; S)$ is defined by

$$
\xi^{2}(T ; S)=\frac{\mu_{2}(T ; S)}{4 \chi(T ; S)} .
$$

The second field-derivative of the susceptibility $\chi_{4}(T ; S)$ is defined by

$$
\chi_{4}(T ; S)=\sum_{\vec{x}, \vec{y}, \vec{z}}\langle s(\overrightarrow{0}) s(\vec{x}) s(\vec{y}) s(\vec{z})\rangle_{c} .
$$




\section{DEFINITIONS OF CRITICAL PARAMETERS}

In terms of the asymptotic behavior of these observables, we can now define the critical parameters, amplitudes and exponents that we are going to estimate using HT and LT series.

The spontaneous magnetization has the asymptotic behavior

$$
M^{(-)}(T ; S) \simeq B^{(-)}(S)|t(S)|^{\beta(S)}\left(1+a_{M}^{(-)}(S)|t(S)|^{\theta(S)}+. .\right)
$$

as $t(S) \rightarrow 0-$.

The asymptotic behaviour of the susceptibility as $t(S) \rightarrow 0 \pm$, is expected to be

$$
\chi^{( \pm)}(T ; S) \simeq C^{( \pm)}(S)|t(S)|^{-\gamma(S)}\left(1+a_{\chi}^{( \pm)}(S)|t(S)|^{\theta(S)}+\ldots+b_{\chi}^{( \pm)}(S) t(S)+\ldots\right)
$$

The correlation length

$$
\xi^{( \pm)}(T ; S) \simeq f^{( \pm)}(S)|t(S)|^{-\nu(S)}\left(1+a_{\xi}^{( \pm)}(S)|t(S)|^{\theta(S)}+\ldots+b_{\xi}^{( \pm)}(S) t(S)+\ldots\right)
$$

the specific heat

$$
C_{H}^{( \pm)}(T ; S) / k_{B} \simeq A^{( \pm)}(S) \ln |t(S)|\left(1+a_{C}^{( \pm)}(S)|t(S)|^{\theta(S)}+\ldots+b_{C}^{( \pm)}(S) t(S)+\ldots\right)
$$

and the second field-derivative of the susceptibility $\chi_{4}(K ; S)$

$$
\chi_{4}^{( \pm)}(T ; S) \simeq-C_{4}^{( \pm)}(S)|t(S)|^{-\gamma_{4}(S)}\left(1+a_{4}^{( \pm)}(S)|t(S)|^{\theta(S)}+\ldots+b_{4}^{( \pm)}(S) t(S)+\ldots\right)
$$

have analogous asymptotic behaviours.

Different (universal) critical exponents $\beta(S), \gamma(S), \nu(S), \gamma_{4}(S)$ and different (nonuniversal) critical amplitudes $B^{(-)}(S), C^{( \pm)}(S), f^{( \pm)}(S) \ldots, a_{\chi}^{( \pm)}(S), a_{\xi}^{( \pm)}(S)$, etc. are associated with the various observables. We have reported in such detail our definitions of the critical amplitudes, because they differ significantly from those of other authors and it is necessary to use a consistent normalisation convention when comparing models expected to belong to the same universality class. Let us notice in particular that the estimates reported in the tables of Ref. ${ }^{7}$ for the critical amplitudes of the susceptibility $\chi^{(-)}(u ; S)$ are related to ours by the factor $S^{2}\left(-\ln u_{c}(S)\right)^{\gamma} / u_{c}(S)^{4 S}$. A similar remark applies to the specific heat amplitudes $C^{(-)}(u ; S)$ for which the conversion factor is $1 / u_{c}(S)^{4 S}\left(\ln u_{c}(S)\right)^{2}$. Finally, the magnetization amplitudes of Ref. ${ }^{7}$ should be multiplied by the factor $\left(-\ln u_{c}(S)\right)^{1 / 8} / S$ to agree with ours. Of course, the amplitudes of the conformal field theory considered in the study of Ref. ${ }^{17}$ are not comparable to our series quantities.

As indicated in eqs.(9) - (13), for a given spin $S$, all asymptotic forms are moreover expected $^{18}$ to contain leading non-analytic confluent corrections characterized by the same exponent $\theta(S)$. Higher order corrections are also expected to contain logarithmic ${ }^{18}$ factors. If universality holds, all exponents have to be $S$-independent.

The presence and the value of the confluent exponent has been discussed ${ }^{19,20,21,22,23}$ several times. From RG calculations ${ }^{24}$, both in the $\epsilon$-expansion and in the fixed-dimension approach, it was conjectured that $\theta \simeq 4 / 3$ for the universality class of the two-dimensional Ising 
model. Aharony and Fisher and later Blöte and den Nijs $\operatorname{argued}^{19,22}$ that $a_{\chi}^{( \pm)}(S)=0$ for $S=1 / 2$ and indeed no such correction was revealed by the later very accurate study ${ }^{13,25}$ of the critical asymptotic expansion for $\chi(K ; 1 / 2)$. However, in the absence of more general results, the reliable assessment of the subleading asymptotic critical behaviour remained an open problem when $S>1 / 2$.

\section{ESTIMATES OF UNIVERSAL AMPLITUDE COMBINATIONS}

In terms of $\chi^{(+)}(K ; S), \xi^{(+)}(K ; S)$ and $\chi_{4}^{(+)}(K ; S)$, a "hyper-universal" combination of critical amplitudes denoted by $g_{r}^{(+)}(S)$ and usually called the "dimensionless renormalized coupling constant", can be defined by

$$
g_{r}^{(+)}(S)=-\frac{3 v C_{4}^{(+)}(S)}{8 \pi\left(a f^{(+)}(S)\right)^{2} C^{(+)}(S)^{2}}
$$

Here the normalisation factor $\frac{3}{8 \pi}$ is chosen in order to match the usual field theoretic definition $^{24}$ of $g_{r}^{(+)}(S)$ and $v$ denotes the volume per lattice site, measured in units of the square of a lattice constant. For all lattices one has $v=\sigma a^{2}$, with $a$ the lattice constant. For the triangular lattice, $\sigma=\sqrt{3} / 2$, for the honeycomb lattice $\sigma=3 \sqrt{3} / 4$, and for the kagomé lattice $\sigma=2 / \sqrt{3}$.

We have also studied the hyper-universal combination usually denoted as

$$
R_{\xi}^{(+)}(S)=\left(A^{(+)}(S) / v\right)^{1 / 2}\left(a f^{(+)}(S)\right)
$$

and the Watson combination ${ }^{26}$

$$
R_{C}(S)=A^{(+)}(S) C^{(+)}(S) / B^{(-)}(S)^{2}
$$

The other frequently considered universal combination

$$
R_{4}(S)=C_{4}^{(+)}(S) B^{(-)}(S)^{2} / C^{(+)}(S)^{3}
$$

is not independent of the previous ones, since $R_{4}(S)=-\frac{8}{3 \pi} g_{r}^{(+)}(S) R_{\xi}^{(+)}(S)^{2} / R_{C}(S)$.

All of these quantities are accurately known in the $S=1 / 2$ case. As indicated in Ref. ${ }^{14,25}$, it is known that $A^{(+)}(1 / 2)=\frac{2}{\pi} \ln [\tan (\pi / 8)]^{2} \approx 0.4945385895, C^{(+)}(1 / 2) \approx 0.962581732$, and $B^{(-)}(1 / 2)=2^{5 / 16} \ln [1+\sqrt{2}]^{1 / 8} \approx 1.22240995$. In Refs. ${ }^{23,27}$, we find the very accurate estimates $C_{4}^{(+)}(1 / 2)=4.379095(8)$ and $f^{(+)}(1 / 2) \approx 0.5670683$.

Therefore we can conclude $g_{r}^{(+)}(1 / 2)=1.754364(2), R_{C}(1 / 2) \approx 0.31856939, R_{\xi}^{(+)}(1 / 2) \approx$ 0.39878194 and $R_{4}(1 / 2)=7.336744(10)$.

We have also considered the ratio $C^{(+)}(S) / C^{(-)}(S)$. In Ref. ${ }^{25}$, for $S=1 / 2$, this ratio was computed with arbitrary precision to be $C^{(+)}(1 / 2) / C^{(-)}(1 / 2) \approx 37.693652$.

Finally, we have estimated the ratio $A^{(+)}(S) / A^{(-)}(S)$ for various values of $S$. This ratio equals unity for $S=1 / 2$ by self-duality. This was argued ${ }^{28}$ in greater generality for the $q$-state $(0 \leq q \leq 4)$ Potts model on the square lattice, which, for $q=2$, reduces to the $S=1 / 2$ Ising model.

In what follows, we determine the values of these universal amplitude combinations for $S>1 / 2$. The preliminary part of our series analysis is aimed at estimating the critical temperatures using the expansions of $\chi^{(+)}(K ; S)$ for $S \geq 1$. We employed a variety of methods: 
Zinn-Justin improved-ratio formula ${ }^{29}$, Padé approximants (PA) and inhomogeneous differential approximants $(\mathrm{DA})^{30}$. The best results with DA's were obtained from approximants such that the polynomial coefficient of the highest derivative is even. (As a consequence, the approximants always contain an additional anti-ferromagnetic singularity at $-K_{c}(S)$, beside the one at $K_{c}(S)$ ). Similarly to the LT analysis, but to a much smaller extent, the accuracy of our results tends to deteriorate with increasing $S$. In spite of this, our final HT estimates of the critical points, reported in Table III, show significant improvement in apparent accuracy and sizable discrepancies from the previous LT determinations ${ }^{7}$.

For general values of $S$, less accurate estimates of $K_{c}(S)$ have been obtained in Ref. ${ }^{31}$ from the ten term susceptibility series of Ref. ${ }^{3}$ and from a renormalization group method. More recently other estimates ${ }^{32}$ were obtained by a generalized cluster method. To our knowledge other accurate determinations of the critical points are available only for $S=1$. They have been obtained either by analysing ${ }^{21}$ the 21 term HT series of Ref. ${ }^{6}$ for the susceptibility or by various transfer-matrix methods ${ }^{33,34,35}$. Some of these results have also been cited in Table III.

We have then turned to the critical exponents $\gamma(S), \nu(S)$ and $\gamma_{4}(S)$ and have evaluated them from the log-derivatives of the appropriate HT expansions by first order DA's biased with our HT estimates of the critical temperatures. This computation shows that the relative variation of the exponents is smaller than $\approx 10^{-3}$, in the worst case, for $S$ varying between $1 / 2$ and $\infty$. We report these results in Table IV without further details and simply conclude that universality and hyperscaling appear to be well supported for the leading critical exponents.

It is perhaps also worth noticing that assuming the universality of $\gamma$ we can bias and therefore refine the determination of $K_{c}(S)$. This procedure does not change the central values of the critical points with respect to the unbiased one, but reduces the error bars.

On the other hand, the estimate of the exponent $\theta(S)$ of the leading singular confluent corrections to scaling in the various observables remains quite elusive. Performing either a Baker-Hunter $^{36}$ or a Zinn-Justin ${ }^{29}$ analysis, we can conclude that, at the level of accuracy

TABLE III: Estimates of the critical inverse temperatures for the spin- $S$ Ising models on the sq lattice. Of course, the estimate $K_{c}^{(+)}(S)$, obtained from the HT series, must equal $K_{c}^{(-)}(S)$ obtained from the LT series, and their common value is known exactly only for $S=1 / 2$. For comparison, we have also reported other results beside those obtained from our HT and those obtained in Ref. ${ }^{7}$ from the analysis of LT series. No error estimates are provided in Ref. ${ }^{31}$ for the estimates of $K_{c}^{(+)}(S)$ obtained from the ten term series ${ }^{3}$ of Camp and Van Dyke as well as for the estimates $K_{c}(S)$ obtained by a renormalization group method.

\begin{tabular}{lccccccc}
\hline \hline & $\mathrm{S}=1 / 2$ & $\mathrm{~S}=1$ & $\mathrm{~S}=3 / 2$ & $\mathrm{~S}=2$ & $\mathrm{~S}=5 / 2$ & $\mathrm{~S}=3$ & $\mathrm{~S}=\infty$ \\
\hline$K_{c}^{(+)}(S)$ & $0.44068679 \ldots$ & $0.590473(5)$ & $0.684255(6)$ & $0.748562(8)$ & $0.79541(1)$ & $0.83106(2)$ & $1.09315(2)$ \\
$K_{c}^{(-)}(S)^{7}$ & $0.44068679 \ldots$ & $0.5904727(9)$ & $0.684338(46)$ & $0.7487(14)$ & $0.8025(35)$ & $0.839(10)$ & \\
$K_{c}^{(+)}(S)^{31}$ & 0.441 & 0.592 & 0.687 & 0.752 & 0.800 & 0.836 \\
$K_{c}(S)^{31}$ & 0.458 & 0.610 & 0.704 & 0.770 & 0.818 & 0.855 \\
$K_{c}^{(+)}(S)^{33}$ & \multicolumn{7}{c}{$0.5904727(10)$} \\
$K_{c}(S)^{35}$ & 0.590471 & & & \\
$K_{c}(S)^{34}$ & $0.590076 \ldots$ & & & \\
\hline \hline
\end{tabular}


TABLE IV: Estimates of critical exponents obtained from our HT series for the spin- $S$ Ising models on the sq lattice. Of course the values of $\gamma, \nu$ and $\gamma_{4}$, for $S=1 / 2$ are exactly known.

\begin{tabular}{lccccccc}
\hline \hline & $\mathrm{S}=1 / 2$ & $\mathrm{~S}=1$ & $\mathrm{~S}=3 / 2$ & $\mathrm{~S}=2$ & $\mathrm{~S}=5 / 2$ & $\mathrm{~S}=3$ & $\mathrm{~S}=\infty$ \\
\hline$\gamma(S)$ & 1.75 & $1.7502(4)$ & $1.7500(4)$ & $1.7496(5)$ & $1.7500(5)$ & $1.7501(5)$ & $1.7494(8)$ \\
$\nu(S)$ & 1.0 & $0.9999(6)$ & $0.9996(8)$ & $0.9994(8)$ & $0.9994(8)$ & $0.9994(8)$ & $0.9994(8)$ \\
$\gamma_{4}(S)$ & 5.5 & $5.498(4)$ & $5.497(5)$ & $5.497(5)$ & $5.497(5)$ & $5.497(5)$ & $5.497(5)$ \\
\hline \hline
\end{tabular}

made possible by the present extension of the HT series, the amplitudes of these corrections are very small, (or perhaps vanishing) for all values of $S$. We should mention that a similar conclusion was suggested for $S=1$ in Ref. ${ }^{22}$, while the opposite conclusion was advocated in Ref. ${ }^{21}$.

Once we have estimated the critical temperatures and verified the universality of the leading exponents, we can proceed with the analysis simply assuming that, for all values of $S$, these exponents take exactly the values expected for the universality class of the two-dimensional spin-1/2 Ising model and using them along with our estimated critical temperatures to bias the evaluation of the HT and LT critical amplitudes defined by eqs.(9) - (13).

Our estimates of the critical amplitudes are reported in Table V.

We have employed quasi-diagonal non-defective PA's or DA's for extrapolating to $K_{c}(S)$ the effective amplitudes of the susceptibility and of the derivative of the specific heat, from the HT and the LT side of the critical points. We have similarly studied the effective amplitudes of the correlation-length (available only in the HT region) and of the magnetization. For proper comparison, in the same Table we have also cited the LT estimates of the critical amplitudes for the spontaneous magnetization, the specific heat and the susceptibility previously obtained in Ref. ${ }^{7}$. These quantities have been multiplied by the above indicated

TABLE V: Our series estimates of the critical amplitudes on the HT and the LT side of the critical point for various spin- $S$ Ising models on the square lattice. Along with our estimates of the LT amplitudes we have reported for comparison also the results of Ref. ${ }^{7}$ multiplied by the proper conversion factors. The values of $C^{(+)}(1 / 2), A^{(+)}(1 / 2), C^{(-)}(1 / 2), B^{(-)}(1 / 2)$ and $A^{(-)}(1 / 2)$ are exactly known, those of $f^{(+)}(1 / 2)$ and $C_{4}^{(+)}(1 / 2)$ are very accurately known.

\begin{tabular}{lccccccc}
\hline \hline & $\mathrm{S}=1 / 2$ (ex.) & $\mathrm{S}=1$ & $\mathrm{~S}=3 / 2$ & $\mathrm{~S}=2$ & $\mathrm{~S}=5 / 2$ & $\mathrm{~S}=3$ & $\mathrm{~S}=\infty$ \\
\hline$C^{(+)}(S)$ & $0.9625817 \ldots$ & $0.5514(2)$ & $0.4307(2)$ & $0.3755(2)$ & $0.3441(2)$ & $0.3254(3)$ & $0.2351(2)$ \\
$A^{(+)}(S)$ & $0.494538589 \ldots$ & $0.736(10)$ & $0.854(4)$ & $0.917(4)$ & $0.956(4)$ & $0.983(4)$ & $1.054(6)$ \\
$f^{(+)}(S)$ & $0.567068 \ldots$ & $0.4640(2)$ & $0.4309(2)$ & $0.4159(2)$ & $0.4082(2)$ & $0.4033(2)$ & $0.3900(2)$ \\
$C_{4}^{(+)}(S)$ & $4.379095(8)$ & $0.9630(4)$ & $0.5073(3)$ & $0.3591(3)$ & $0.2902(3)$ & $0.2533(3)$ & $0.1239(3)$ \\
\hline$C^{(-)}(S)$ & $0.02553697 \ldots$ & $0.01462(3)$ & $0.0114(3)$ & $0.0102(6)$ & $0.0090(8)$ & $0.0055(30)$ \\
$C^{(-)}(S)^{7}$ & & $0.01462(2)$ & $0.0109(29)$ & $0.0094(10)$ & $0.0096(33)$ & & \\
\hline$B^{(-)}(S)$ & $1.22240995 \ldots$ & $1.131(4)$ & $1.076(5)$ & $1.041(5)$ & $1.016(5)$ & $1.001(5)$ & \\
$B^{(-)}(S)^{7}$ & & $1.1313(2)$ & $1.077(9)$ & $1.042(16)$ & $1.030(19)$ & $1.016(26)$ & \\
\hline$A^{(-)}(S)$ & $0.494538589 \ldots$ & $0.738(6)$ & $0.855(10)$ & $0.915(10)$ & $1.1(2)$ & $1.1(2)$ & \\
$A^{(-)}(S)^{7}$ & & $0.73(2)$ & $0.77(6)$ & $0.86(8)$ & $0.87(9)$ & & \\
\hline \hline
\end{tabular}


TABLE VI: Universal combinations of critical amplitudes for various spin- $S$ Ising models on the square lattice. The exactly (or very accurately) known values for $S=1 / 2$ are reported in the first column. For $S>1 / 2$, the series estimates of this note are reported in the successive columns. In the last line we have reported the estimates of $R_{C}(S)$ obtained by combining our present estimates of $A^{(+)}(S)$ and $C^{(+)}(S)$ with the estimates of $B^{(-)}(S)$ given in Ref. ${ }^{7}$.

\begin{tabular}{lccccccc}
\hline \hline & $\mathrm{S}=1 / 2($ ex. $)$ & $\mathrm{S}=1$ & $\mathrm{~S}=3 / 2$ & $\mathrm{~S}=2$ & $\mathrm{~S}=5 / 2$ & $\mathrm{~S}=3$ & $S=\infty$ \\
\hline$C^{(+)}(S) / C^{(-)}(S)$ & $37.693652 \ldots$ & $37.71(9)$ & $38(1)$. & $37(2)$. & $38(3)$. & $59(32)$. & \\
$A^{(+)}(S) / A^{(-)}(S)$ & 1.0 & $0.997(21)$ & $0.999(16)$ & $1.0(1)$ & $0.87(16)$ & $0.89(17)$ & \\
$g_{r}^{(+)}(S)$ & $1.754364(2)$ & $1.753(2)$ & $1.753(2)$ & $1.752(3)$ & $1.752(3)$ & $1.752(3)$ & $1.752(3)$ \\
$R_{\xi}^{(+)}(S)$ & $0.39878194 \ldots$ & $0.398(3)$ & $0.398(1)$ & $0.398(1)$ & $0.399(1)$ & $0.400(2)$ & $0.400(2)$ \\
$R_{C}(S)$ & $0.31856939 \ldots$ & $0.317(5)$ & $0.318(2)$ & $0.318(2)$ & $0.319(3)$ & $0.319(5)$ & \\
$R_{C}(S)^{7}$ & & $0.317(4)$ & $0.317(7)$ & $0.317(11)$ & $0.31(1)$ & $0.31(1)$ & \\
\hline \hline
\end{tabular}

conversion factors to agree with our normalisation conventions. The uncertainties we have reported, which allow for the observed spreads in the approximant values, provide a subjective assessment of residual trends in the sequence of estimates and for the (unbiased) uncertainties of the critical points. The HT amplitudes can be determined with a relative accuracy ranging from $\approx 10^{-3}$ in the case of the susceptibility, to $\approx 10^{-2}$ in the case of the specific heat. The LT amplitudes are subject to larger relative uncertainties, increasing with $S$, and reaching up to $\approx 50 \%$ for $S>2$. In some cases, in order to improve the accuracy of the estimates of the LT amplitudes for $S \leq 2$, we have based our extrapolations only on the data for $|t(S)| \gtrsim 0.02-0.04$. This unconventional but reasonable procedure reduces the sensitivity of the approximants to the unphysical nearby singularities. Unfortunately, even this prescription fails to work satisfactorily for $S>2$.

Using only the HT series, we can evaluate $g_{r}^{(+)}(S)$, either directly, in terms of the amplitudes reported in Table $\mathrm{V}$, or by extrapolating to the critical points via DA's the HT expansion of the inverse effective coupling $1 / g_{r}^{(+)}(K ; S)=-\frac{8 \pi}{3} \xi(K ; S)^{2} \chi(K ; S)^{2} / \chi_{4}(K ; S)$. A third approach consists in studying the residua at $x=1$ of the series with coefficients $a_{n}(S)=c_{n}(S) / d_{n}(S)$, where $c_{n}(S)$ are the HT coefficients of $\xi^{2}(K ; S)$ and $d_{n}(S)$ are the coefficients of the quantity $\chi_{4}(K ; S) / \chi^{2}(K ; S)$. In Table $\mathrm{V}$ we have reported the results of the latter procedure since it yields estimates with smaller spreads.

Several other estimates ${ }^{23,37,38,39,40,41}$ obtained by a variety of methods are also available in the literature.

Using also the LT series, we have evaluated, directly in terms of the single amplitudes, the other mentioned universal combinations, for a range of values of $S$. We have reported in Table VI, our series estimates of all these quantities for $S>1 / 2$. In conclusion, whenever only HT amplitudes are involved, our estimates, within a precision up to $0.1 \%$, are independent of $S$, in full agreement with universality. On the other hand, our reanalysis of the LT series has been only partially successful: whenever LT amplitudes also enter into the combinations, universality appears to be fairly well respected for $S<5 / 2$, but the uncertainties grow notably larger for larger values of the spin.

In the next section we describe the theory of lattice-lattice scaling, and show how it can be used to extend our estimates of the critical amplitudes from the square lattice to other two-dimensional lattices. 


\section{LATTICE-LATTICE SCALING}

The theory of lattice-lattice scaling was developed by Betts, Guttmann and Joyce ${ }^{15}$ in the early '70s. It explains how amplitudes change within a given universality class, as one moves from one lattice to another. It can also be viewed as a generalisation of the law of corresponding states. In this section we give a terse development of the theory, and apply it to the problem at hand.

In order to review the general ideas let us first consider the Weiss theory or mean field theory of a magnetic system. The equation of state is well known to be

$$
h=\frac{1}{3} m^{3}\left(1+3 t / m^{2}\right) .
$$

Here $t=T / T_{c}-1, h=\mu H / k T$, and $m=M(T) / M(0)$ are the reduced temperature, magnetic field and magnetization, respectively.

Then the law of corresponding states says that the equation of state is the same for all lattices. That is,

$$
m_{\mathrm{X}}(t, h)=m_{\mathrm{Y}}(t, h),
$$

where $\mathrm{X}$ and $\mathrm{Y}$ denote two lattices. That is to say, the lattice dependence is entirely contained in the critical temperature $T_{c}$.

A more complex model is the three-dimensional spherical model, for which the critical equation of state is:

$$
h=D_{\mathrm{X}} m^{5}\left(1+t / m^{2}\right)^{2} .
$$

Here both $T_{c}$ and the amplitude $D$ are lattice dependent. Thus

$$
m_{\mathrm{X}}\left(t, h_{\mathrm{X}}\right)=m_{\mathrm{Y}}\left(t, h_{\mathrm{Y}}\right) .
$$

We see that we must scale the field variable, so that $\frac{h_{\mathrm{X}}}{D_{\mathrm{X}}}=\frac{h_{\mathrm{Y}}}{D_{\mathrm{Y}}}$, but that there is no need to scale the reduced temperature.

Let us now consider the case of the (zero-field) spin $S=1 / 2$ Ising model on the triangular $(\mathrm{T})$ and hexagonal $(\mathrm{H})$ lattices. The star-triangle relation ${ }^{42,43}$ allows us to relate the freeenergy, susceptibility and spontaneous magnetization between the lattices:

$$
\begin{array}{r}
2 f_{\mathrm{H}}\left(K_{\mathrm{H}}\right)=f_{\mathrm{T}}\left(K_{\mathrm{T}}\right), \\
M_{\mathrm{T}, 0}\left(t_{\mathrm{T}}\right)=M_{\mathrm{H}, 0}\left(t_{\mathrm{H}}\right), \\
2 \chi_{\mathrm{T}}\left(v_{\mathrm{T}}\right)=\chi_{\mathrm{H}}\left(v_{\mathrm{H}}\right)+\chi_{\mathrm{H}}\left(-v_{\mathrm{H}}\right),
\end{array}
$$

where $f=1 / N \ln Z, K_{\mathrm{X}}=J_{\mathrm{X}} / k T$, and $v_{\mathrm{X}}=\tanh \left(J_{\mathrm{X}} / k T\right)$. Here we see that the reduced temperature needs to be re-scaled for the free-energy to be universal. This is not restricted to the triangular-honeycomb pair, but in that case it is easy to be explicit.

All these examples can be encapsulated in the following expression for the singular part of the free-energy:

$$
n_{\mathrm{X}} f_{\mathrm{X}}\left(t_{\mathrm{X}}, h_{\mathrm{X}}\right)=n_{\mathrm{Y}} f_{\mathrm{Y}}\left(t_{\mathrm{Y}}, h_{\mathrm{Y}}\right)=f(t, h),
$$

where the reduced temperature and field are scaled by

$$
n_{\mathrm{X}} h_{\mathrm{X}}=n_{\mathrm{Y}} h_{\mathrm{Y}}=h
$$

and

$$
g_{\mathrm{X}} t_{\mathrm{X}}=g_{\mathrm{Y}} t_{\mathrm{Y}}=t
$$


The singular part of the free-energy, $f(t, h)$, is then a universal (lattice independent) function for a given model.

Equivalently, by differentiation we obtain

$$
m_{\mathrm{X}}\left(t_{\mathrm{X}}, h_{\mathrm{X}}\right)=m_{\mathrm{Y}}\left(t_{\mathrm{Y}}, h_{\mathrm{Y}}\right)=m(t, h),
$$

and

$$
\chi_{\mathrm{X}}\left(t_{\mathrm{X}}, h_{\mathrm{X}}\right) / n_{\mathrm{X}}=\chi_{\mathrm{Y}}\left(t_{\mathrm{Y}}, h_{\mathrm{Y}}\right) / n_{\mathrm{Y}}=\chi(t, h),
$$

where $m(t, h)$ and $\chi(t, h)$ are universal functions for a given model.

Writing $m_{\mathrm{X}}=B_{\mathrm{X}}^{(-)}\left(-t_{\mathrm{X}}\right)^{\beta}$, it follows that

$$
\frac{g_{\mathrm{X}}}{g_{\mathrm{Y}}}=\left(\frac{B_{\mathrm{X}}^{(-)}}{B_{\mathrm{Y}}^{(-)}}\right)^{1 / \beta} .
$$

Using this result, and the exact scaling parameters $g_{\mathrm{X}}$ and $g_{\mathrm{Y}}$ given below, it is a trivial matter to calculate the magnetization amplitudes for the other lattices we consider (triangular, hexagonal and kagomé $(\mathrm{K})$ ), taking as input the square lattice amplitudes given in Table V. These amplitude estimates are given in Tables VII, VIII and IX.

Writing $\chi_{\mathrm{X}}=C_{\mathrm{X}}^{( \pm)} t_{\mathrm{X}}^{-\gamma}$ it follows that

$$
C_{\mathrm{X}}^{(+)} / C_{\mathrm{Y}}^{(+)}=\frac{n_{\mathrm{X}}}{n_{\mathrm{Y}}}\left(\frac{g_{\mathrm{X}}}{g_{\mathrm{Y}}}\right)^{-\gamma}=C_{\mathrm{X}}^{(-)} / C_{\mathrm{Y}}^{(-)} .
$$

Similarly, it is a trivial matter to calculate the susceptibility amplitudes for the other lattices we consider, taking as input the $S=1 / 2$ square lattice amplitudes given in Table V.

Further differentiation gives the corresponding relationship for higher field derivatives, and we readily obtain

$$
C_{2 l, \mathrm{X}}^{(+)} / C_{2 l, \mathrm{Y}}^{(+)}=\left(\frac{n_{\mathrm{X}}}{n_{\mathrm{Y}}}\right)^{2 l-1}\left(\frac{g_{\mathrm{X}}}{g_{\mathrm{Y}}}\right)^{-\gamma_{2 l}},
$$

for the high-temperature field derivatives, (where only the even-order derivatives are nonzero). The corresponding result for low-temperature field derivatives is

$$
C_{l, \mathrm{X}}^{(-)} / C_{l, \mathrm{Y}}^{(-)}=\left(\frac{n_{\mathrm{X}}}{n_{\mathrm{Y}}}\right)^{l-1}\left(\frac{g_{\mathrm{X}}}{g_{\mathrm{Y}}}\right)^{-\gamma_{l}^{\prime}} .
$$

Taking temperature derivatives, one readily establishes that the specific heat amplitudes satisfy

$$
A_{\mathrm{X}}^{(+)} / A_{\mathrm{Y}}^{(+)}=\frac{n_{\mathrm{Y}}}{n_{\mathrm{X}}}\left(\frac{g_{\mathrm{X}}}{g_{\mathrm{Y}}}\right)^{2-\alpha}=A_{\mathrm{X}}^{(-)} / A_{\mathrm{Y}}^{(-)} .
$$

As $\alpha=0$ for the two-dimensional Ising model, this simplifies to

$$
A_{\mathrm{X}}^{(+)} / A_{\mathrm{Y}}^{(+)}=\frac{n_{\mathrm{Y}}}{n_{\mathrm{X}}}\left(\frac{g_{\mathrm{X}}}{g_{\mathrm{Y}}}\right)^{2}=A_{\mathrm{X}}^{(-)} / A_{\mathrm{Y}}^{(-)} .
$$

We have similarly calculated the specific-heat amplitudes for the other lattices we consider, taking as input the square lattice amplitudes given in Table VI. These are also given in Tables VII, VIII, and IX. 
TABLE VII: Estimates of the critical amplitudes on the HT and the LT side of the critical point for various spin- $S$ Ising models on the triangular lattice, as obtained by lattice-lattice scaling, using the square-lattice series estimates as a basis. The values of $C^{(+)}(1 / 2), A^{(+)}(1 / 2), C^{(-)}(1 / 2)$, $B^{(-)}(1 / 2)$ and $A^{(-)}(1 / 2)$ are exactly known.

\begin{tabular}{lccccccc}
\hline \hline & $\mathrm{S}=1 / 2(\mathrm{ex})$. & $\mathrm{S}=1$ & $\mathrm{~S}=3 / 2$ & $\mathrm{~S}=2$ & $\mathrm{~S}=5 / 2$ & $\mathrm{~S}=3$ & $\mathrm{~S}=\infty$ \\
\hline$C^{(+)}(S)$ & $0.92420696 \ldots$ & $0.5294(2)$ & $0.4135(2)$ & $0.3605(2)$ & $0.3304(2)$ & $0.3124(3)$ & $0.2257(2)$ \\
$A^{(+)}(S)$ & $0.499069377 \ldots$ & $0.743(10)$ & $0.862(4)$ & $0.925(4)$ & $0.965(4)$ & $0.992(4)$ & $1.064(6)$ \\
$f^{(+)}(S)$ & $0.525315 \ldots$ & $0.4298(2)$ & $0.3992(2)$ & $0.3853(2)$ & $0.3781(2)$ & $0.3736(2)$ & $0.3613(2)$ \\
$C_{4}^{(+)}(S)$ & $4.000248(8)$ & $0.8797(4)$ & $0.4634(3)$ & $0.3280(3)$ & $0.2651(3)$ & $0.2314(3)$ & $0.1132(3)$ \\
\hline$C^{(-)}(S)$ & $0.024518902 \ldots$ & $0.01404(3)$ & $0.0109(3)$ & $0.0098(6)$ & $0.0086(8)$ & $0.0053(30)$ & \\
\hline$B^{(-)}(S)$ & $1.203269903 \ldots$ & $1.113(4)$ & $1.059(5)$ & $1.025(5)$ & $1.000(5)$ & $0.985(5)$ & \\
\hline$A^{(-)}(S)$ & $0.4990693724 \ldots$ & $0.745(6)$ & $0.863(10)$ & $0.923(10)$ & $1.1(2)$ & $1.1(2)$ & \\
\hline \hline
\end{tabular}

TABLE VIII: Estimates of the critical amplitudes on the HT and the LT side of the critical point for various spin- $S$ Ising models on the hexagonal lattice, as obtained by lattice-lattice scaling, using the square-lattice series estimates as a basis. The values of $C^{(+)}(1 / 2), A^{(+)}(1 / 2), C^{(-)}(1 / 2)$, $B^{(-)}(1 / 2)$ and $A^{(-)}(1 / 2)$ are exactly known.

\begin{tabular}{lccccccc}
\hline \hline & $\mathrm{S}=1 / 2($ ex. $)$ & $\mathrm{S}=1$ & $\mathrm{~S}=3 / 2$ & $\mathrm{~S}=2$ & $\mathrm{~S}=5 / 2$ & $\mathrm{~S}=3$ & $\mathrm{~S}=\infty$ \\
\hline$C^{(+)}(S)$ & $1.0464170 \ldots$ & $0.5994(2)$ & $0.4682(2)$ & $0.4082(2)$ & $0.3741(2)$ & $0.3537(3)$ & $0.2556(2)$ \\
$A^{(+)}(S)$ & $0.4781063817 \ldots$ & $0.712(10)$ & $0.826(4)$ & $0.887(4)$ & $0.924(4)$ & $0.950(4)$ & $1.019(6)$ \\
$f^{(+)}(S)$ & $0.657331 \ldots$ & $0.5379(2)$ & $0.4995(2)$ & $0.4821(2)$ & $0.4732(2)$ & $0.4675(2)$ & $0.4521(2)$ \\
$C_{4}^{(+)}(S)$ & $5.352965(8)$ & $1.1772(4)$ & $0.6201(3)$ & $0.4390(3)$ & $0.3547(3)$ & $0.3096(3)$ & $0.1515(3)$ \\
\hline$C^{(-)}(S)$ & $0.027761095 \ldots$ & $0.01589(3)$ & $0.0124(3)$ & $0.0111(6)$ & $0.0098(8)$ & $0.0060(30)$ & \\
\hline$B^{(-)}(S)$ & $1.253177691 \ldots$ & $1.159(4)$ & $1.103(5)$ & $1.067(5)$ & $1.042(5)$ & $1.026(5)$ & \\
\hline$A^{(-)}(S)$ & $0.4781063817 \ldots$ & $0.714(6)$ & $0.827(10)$ & $0.885(10)$ & $1.1(2)$ & $1.1(2)$ & \\
\hline \hline
\end{tabular}

TABLE IX: Estimates of the critical amplitudes on the HT and the LT side of the critical point for various spin- $S$ Ising models on the kagomé lattice, as obtained by lattice-lattice scaling, using the square-lattice series estimates as a basis. The values of $C^{(+)}(1 / 2), A^{(+)}(1 / 2), C^{(-)}(1 / 2), B^{(-)}(1 / 2)$ and $A^{(-)}(1 / 2)$ are exactly known.

\begin{tabular}{lccccccc}
\hline \hline & $\mathrm{S}=1 / 2($ ex. $)$ & $\mathrm{S}=1$ & $\mathrm{~S}=3 / 2$ & $\mathrm{~S}=2$ & $\mathrm{~S}=5 / 2$ & $\mathrm{~S}=3$ & $\mathrm{~S}=\infty$ \\
\hline$C^{(+)}(S)$ & $1.01814223 \ldots$ & $0.5832(2)$ & $0.4556(2)$ & $0.3972(2)$ & $0.3640(2)$ & $0.3442(3)$ & $0.2487(2)$ \\
$A^{(+)}(S)$ & $0.4800615653 \ldots$ & $0.714(10)$ & $0.829(4)$ & $0.890(4)$ & $0.928(4)$ & $0.954(4)$ & $1.023(6)$ \\
$f^{(+)}(S)$ & $0.618474 \ldots$ & $0.5061(2)$ & $0.4700(2)$ & $0.4536(2)$ & $0.4452(2)$ & $0.4399(2)$ & $0.4254(2)$ \\
$C_{4}^{(+)}(S)$ & $5.046953(8)$ & $1.1099(4)$ & $0.5847(3)$ & $0.4139(3)$ & $0.3345(3)$ & $0.2919(3)$ & $0.1428(3)$ \\
\hline$C^{(-)}(S)$ & $0.02701097 \ldots$ & $0.01546(3)$ & $0.0121(3)$ & $0.0108(6)$ & $0.0095(8)$ & $0.0058(30)$ & \\
\hline$B^{(-)}(S)$ & $1.238655888 \ldots$ & $1.146(4)$ & $1.090(5)$ & $1.055(5)$ & $1.030(5)$ & $1.014(5)$ & \\
\hline$A^{(-)}(S)$ & $0.4800615653 \ldots$ & $0.716(6)$ & $0.830(10)$ & $0.888(10)$ & $1.1(2)$ & $1.1(2)$ & \\
\hline \hline
\end{tabular}


Finally, the correlation function amplitudes were calculated exactly from various startriangle transformations by Thompson and Guttmann ${ }^{44}$ for the true correlation length. It follows from universality that these same transformations should hold also for the secondmoment correlation length amplitudes. We show below that this is equivalent to the universality of $R_{\xi}^{(+)}(S)$, which is a conclusion of this work.

$\mathrm{In}^{44}$ it was shown by explicit calculation that

$$
\begin{aligned}
& f_{\mathrm{T}}^{(+)}(1 / 2) K_{c, \mathrm{~T}}^{(+)}(1 / 2) / f_{\mathrm{H}}^{(+)}(1 / 2) K_{c, \mathrm{H}}^{(+)}(1 / 2)=1 / 3 . \\
& f_{\mathrm{S}}^{(+)}(1 / 2) K_{c, \mathrm{~S}}^{(+)}(1 / 2) / f_{\mathrm{T}}^{(+)}(1 / 2) K_{c, \mathrm{~T}}^{(+)}(1 / 2)=\sqrt{3} . \\
& f_{\mathrm{K}}^{(+)}(1 / 2) K_{c, \mathrm{~K}}^{(+)}(1 / 2) / f_{\mathrm{H}}^{(+)}(1 / 2) K_{c, \mathrm{H}}^{(+)}(1 / 2)=2 / 3 .
\end{aligned}
$$

The universality of $R_{\xi}^{(+)}(S)=\left(\frac{A^{+}(S)}{v}\right)^{1 / 2} a f^{+}(S)$, taken together with the above latticelattice scaling relation for the specific heat amplitude $A^{+}(S)$ implies the lattice-lattice scaling relation

$$
f_{\mathrm{X}}^{(+)} / f_{\mathrm{Y}}^{(+)}=\frac{g_{\mathrm{Y}}}{g_{\mathrm{X}}}\left(\frac{\sigma_{\mathrm{X}} n_{\mathrm{X}}}{\sigma_{\mathrm{Y}} n_{\mathrm{Y}}}\right)^{1 / 2},
$$

where $\sigma$ is the area per site, and $\sigma_{\mathrm{X}}=1, \sqrt{3} / 2,3 \sqrt{3} / 4$ and $2 / \sqrt{3}$ for the square, triangular, honeycomb and kagomé lattices respectively. This is equivalent to the explicit amplitude scaling reported $\mathrm{in}^{44}$, and confirms the expectation that the true correlation length amplitude and the second-moment correlation length amplitude scale similarly.

For the $2 \mathrm{~d}$ Ising model we can calculate the scaling parameters $n_{\mathrm{X}}$ and $g_{\mathrm{X}}$ exactly from known spin-1/2 spontaneous magnetization and specific heat amplitudes. The critical points are also exactly known. These are given in the table below:

$\begin{array}{llll}\mathrm{X} & K_{c} & n_{\mathrm{X}} & g_{\mathrm{X}} K_{c, \mathrm{~T}} / K_{c, \mathrm{X}} \\ \text { Tr. } & \ln (3) / 4 & 1 & 1 \\ \text { Sq. } & -\ln (\sqrt{2}-1) / 2 & 3 \sqrt{3} / 4 & 1 / \sqrt{2} \\ \text { Ho. } & -\ln (2-\sqrt{3}) / 2 & 2 & 1 / \sqrt{3} \\ \text { Ka. } & -\ln (2 / \sqrt{3}-1) / 4 & 3^{5}(\sqrt{3}-1)^{16} & 9(\sqrt{3}-1)^{8}\end{array}$

In addition to the above results, one can also derive scaling relations for the amplitudes of sub-dominant singularities. For example, consider the susceptibility of the $2 \mathrm{~d}$ Ising model on lattice X. Writing

$$
\chi_{\mathrm{X}} \sim C_{0}^{\mathrm{X}} t^{-\gamma}+C_{1}^{\mathrm{X}} t^{1-\gamma},
$$

from lattice-lattice scaling we can derive the following amplitude relations:

$$
\begin{gathered}
\frac{C_{0}^{\mathrm{X}}}{C_{0}^{\mathrm{T}}}=\frac{n_{\mathrm{X}}}{n_{\mathrm{Y}}}\left(\frac{g_{\mathrm{Y}}}{g_{\mathrm{X}}}\right)^{\gamma}, \\
\frac{C_{1}^{\mathrm{X}}}{C_{1}^{\mathrm{T}}}=\frac{n_{\mathrm{X}}}{n_{\mathrm{Y}}}\left(\frac{g_{\mathrm{Y}}}{g_{\mathrm{X}}}\right)^{\gamma-1} .
\end{gathered}
$$

The second expression is false for the kagomé lattice. It is corrected by the theory of extended lattice-lattice scaling developed by Gaunt and Guttmann ${ }^{16}$ in 1978. In that theory, a third scaling parameter needs to be introduced. 
It is widely accepted, and in complete agreement with the results of the first part of this paper, that the spin- $S$ Ising model is in the same universality class as the spin- $1 / 2$ Ising model. This is the only assumption we require in order to apply the theory of lattice-lattice scaling to the square lattice data given in the previous section. The only subtlety is whether "universality" really extends to lattice-lattice universality. While this assumption seems natural, we did attempt to verify it by estimating amplitudes for other lattices from the rather short data available $\mathrm{in}^{3}$. The longest effective series is the triangular lattice series. We found the high-temperature susceptibility amplitude, as estimated by Padé approximants, to be $C^{(+)}(1)=0.529$ from this series, in complete agreement with the more precise value $C^{(+)}(1)=0.5294(2)$ found by lattice-lattice scaling, and reported in Table VII.

Note too that there is no loss of accuracy, as the conversions from lattice to lattice are exact. For example, based on the recent estimate of the leading susceptibility amplitude of the spin $1 / 2$ square lattice Ising model given $\mathrm{in}^{13}$, application of lattice-lattice scaling gives the corresponding amplitude on the triangular lattice to the same precision, viz:

$$
C_{+}^{T}=0.9242069582451643296971575778559317176696261520028389 .
$$

Similarly accurate results for other lattices can be readily written down, as can equally accurate sub-dominant amplitudes.

\section{Acknowledgments}

This work was completed before the untimely death of Marco Comi, our lifelong friend and coauthor. To his dear memory we dedicate this paper.

This work has been partially supported by the Ministry of Education, University and Research (PB, MC), and the Australian Research Council (AJG).

* Electronic address: butera@mib.infn.it

$\dagger$ Electronic address: tonyg@ms.unimelb.edu.au

1 C. Domb and M. F. Sykes, Phys. Rev. 128, 168 (1962).

2 M. A. Moore and J. E. T. Trainin, J. Phys. C 5, L9 (1972).

3 W. J. Camp and J. P. Van Dyke, Phys. Rev. B 11, 2579 (1975).

4 P. F. Fox and A. J. Guttmann, J. Phys. C 6, 913 (1973).

5 B. G. Nickel, in Phase Transitions: Cargese 1980, edited by M. Levy, J. C. Le Guillou and J. Zinn-Justin, (Plenum, New York, 1982); S. Gartenhaus and W. S. McCullough, Phys. Rev. B 38, 11688 (1988).

6 B. G. Nickel and J. J. Rehr, J. Stat. Phys. 61, 1 (1990).

7 I. Jensen, A. J. Guttmann, and I. G. Enting, J. Phys. A 29, 3805 (1996); I. G. Enting, A. J. Guttmann and I. Jensen, J. Phys. A 27, 6987 (1994).

8 G. A. Baker and J. M. Kincaid, J. Stat. Phys. 24, 469 (1981).

9 M. E. Fisher, in Lectures in Theoretical Physics Vol. 7C (Univ. of Colorado Press, Boulder CO, 1965.

10 V. Matveev and R. Shrock, Phys. Lett. A 204, 353 (1995); J. Phys. A 28, L533 (1995).

11 P. Butera and M. Comi, Phys. Rev. B 65, 144431 (2002); hep-lat/0112049. 
12 P. Butera and M. Comi, hep-lat/0204007, only partially published in J. Stat. Phys.109, 311 (2002).

13 B. G. Nickel, J. Phys. A 32, 3889 (1999); W. P. Orrick, B. G. Nickel, A. J. Guttmann and J. H. H. Perk, Phys. Rev. Lett. 86, 4120 (2001). The expansion coefficients computed in this paper through order 323 for the $S=1 / 2$ susceptibility can be found at the URL: http://www.ms.unimelb.edu.au/ tonyg/.

14 V. Privman, P. C. Hohenberg and A. Aharony, in Phase Transitions and critical Phenomena, edited by C. Domb and J. Lebowitz, (Academic, New York, 1989) Vol. 14.

15 D. Betts, A. J. Guttmann and G. S. Joyce J. Phys. C, 4, 1994 (1971).

16 D. S. Gaunt and A. J. Guttmann, J. Phys. A 11, 1381 (1978).

17 G. Delfino, Phys. Lett. B 419, 291 (1998).

18 F. Wegner, Phys. Rev. B 5, 4529 (1972).

19 A. Aharony and M. E. Fisher, Phys. Rev. Lett. 45, 679 (1980); Phys. Rev. B 27, 4394 (1983).

20 M. Barma and M. E. Fisher, Phys. Rev. Lett. 53, 1935 (1984); Phys. Rev. B 31, 5954 (1985).

21 J. Adler, J. Phys. A 20, 3419 (1987); J. Adler and I. G. Enting, J. Phys. A 17, 2233 (1984).

22 H. W. J. Blöte and M. P. M. den Nijs, Phys. Rev. B 37, 1766 (1988).

23 M. Caselle, M. Hasenbusch, A. Pelissetto and E. Vicari, J. Phys. A 34, 2923 (2001).

24 J. Zinn-Justin, Quantum field theory and critical phenomena (Clarendon, Oxford, 1989, third edition 1996).

25 E. Barouch, B. M. McCoy and T. T. Wu, Phys. Rev. Lett. 31, 1409 (1973); T. T. Wu, B. M. McCoy, C. A. Tracy and E. Barouch, Phys. Rev. B 13, 316 (1976).

26 P. G. Watson, J. Phys. C 2, 1883 (1969).

27 J. Salas and A. D. Sokal, J. Stat. Phys. 98, 551 (2000).

28 M. Kaufman and D. Andelman, Phys. Rev. B 29, 4010 (1984).

29 J. Zinn-Justin, J. Phys. (France) 42, 783 (1981).

30 A. J. Guttmann, in Phase Transitions and critical Phenomena, edited by C. Domb and J. Lebowitz, (Academic, New York, 1989) Vol. 13.

31 T. W. Burkhardt and R. H. Swendsen, Phys. Rev. B 13, 3071 (1976).

32 J. L. Monroe, Phys. Rev E65, 066129 (2002).

33 H. W. J. Blöte and M. P. Nightingale, Physica A 134, 274 (1985).

34 M. A. Yurischef, hep-lat/0109025, Nucl. Phys. Proc. Suppl. 106, 917 (2002).

35 A. Lipowski and M. Suzuki, J. Phys. Soc. Jap. 61, 4356 (1992).

36 G. A. Baker and D. L. Hunter, Phys. Rev. B 7, 3377 (1973).

37 C. M. Bender and S. Boettcher, Phys. Rev. B 51, 1875 (1995).

38 S. Y. Zinn, S. N. Lai and M. E. Fisher, Phys. Rev. E 54, 1176 (1996).

39 P. Butera and M. Comi, Phys. Rev. B 54, 15828 (1996).

40 J. K. Kim, J. Phys. A 33, 2675 (2000).

41 J. Balog, M. Niedermaier, F. Niedermayer, A. Patrascioiu, E. Seiler and P. Weisz, Nucl. Phys. B 583, 614(2000).

42 L. Onsager, Phys. Rev. 65, 117 (1944).

43 M. E. Fisher, Phys. Rev. 113, 969 (1959).

44 C. J. Thompson and A. J. Guttmann, Phys. Lett., 53A, 315 (1975). 